\title{
Servo control of behavior rhythms
}

\author{
JAMES D. BOISMIER \\ Nebraska Psychiatric Institute, University of Nebraska College of Medicine \\ 602 South 44 th Street, Omaha, Nebraska 68106
}

and

\author{
DEE L. GRAHAM \\ University of Cincinnati, Cincinatti, Ohio 45221
}

\begin{abstract}
A laboratory computer was used to implement a servo-control arrangement wherein environmental feedback to a behaving animal oscillates sinusoidally in real time. The location and period of behavior rhythms generated by the servo-control arrangement covary with the location and period of the sinusoidal command signal.
\end{abstract}

The world we live in has a variety of temporal rhythms to which we and other forms of life are adapted. With a view to an experimental analysis of behavioral adaptations to environmental rhythms, we have devised a computerized servo-control system which may be used to generate behavior rhythms experimentally. The system is reliable, versatile, and easy to use. In this paper, we describe basic elements of the system and report data which bear on its efficacy.

Servo analysis can be used in the study of behavior processes when feedback to the behaving individual can be quantified and the quantity controlled (cf. Bellville, Fleischli, \& Attura, 1968). In the present arrangement, feedback is the probability of reinforcement of a response emitted at time $t$; this probability density (command signal) oscillates sinusoidally in real time according to Equation 1.

$$
f(t)=a+b \sin 2 \pi(t / d) .
$$

Figure 1 shows a flow chart of the computer program logic used to implement this servo-control arrangement. As indicated, the independently manipulable parameters $\{a, b, d\}$ are specified by the operator at the beginning of an experimental trial: these determine the location $(\mathrm{a}=\mathrm{y}$-intercept), amplitude ( $2|\mathrm{~b}|)$, and real-time period (d sec) of the sinusoidal feedback function. During an experimental trial, the computer is programmed to pole a sense line connected to an operandum and, when a response occurs, to determine its time of occurrence, $t$, solve $f(t)=a+b \sin 2 \pi(t / d)$, generate a random measure of probability density, rnd, then operate a relay connected to a reinforcer

Paper presented to the Fourth Annual Meeting of the National Conference on the Use of On-line Computers in Psychology, Boston, Massachusetts, 20 November, 1974. device (e.g., a food dispenser) if and only if $f(t)>$ rnd.

We also program the computer to maintain a record of experimental data according to the following technique. First, a 60-element data array is initialized at zero. Then, during the trial, each d-sec period is partitioned into 60 equal intervals and responses are tabulated by intervals as they occur. At the end of the trial, the 60 -element array thus contains the number of responses emitted during each 60 th of the d-sec period. These response-per-interval frequencies, in turn, are divided by $1 / 60$ th of the total trial time to

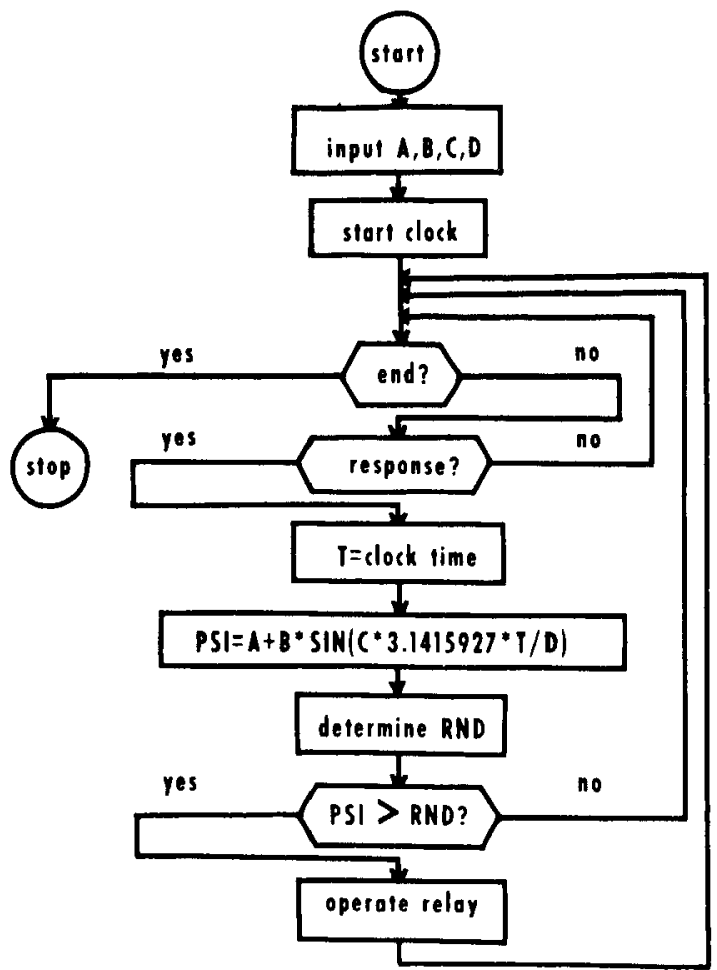

Figure 1. A flow chart of the computer program logic used to implement the servo-control arrangement. 

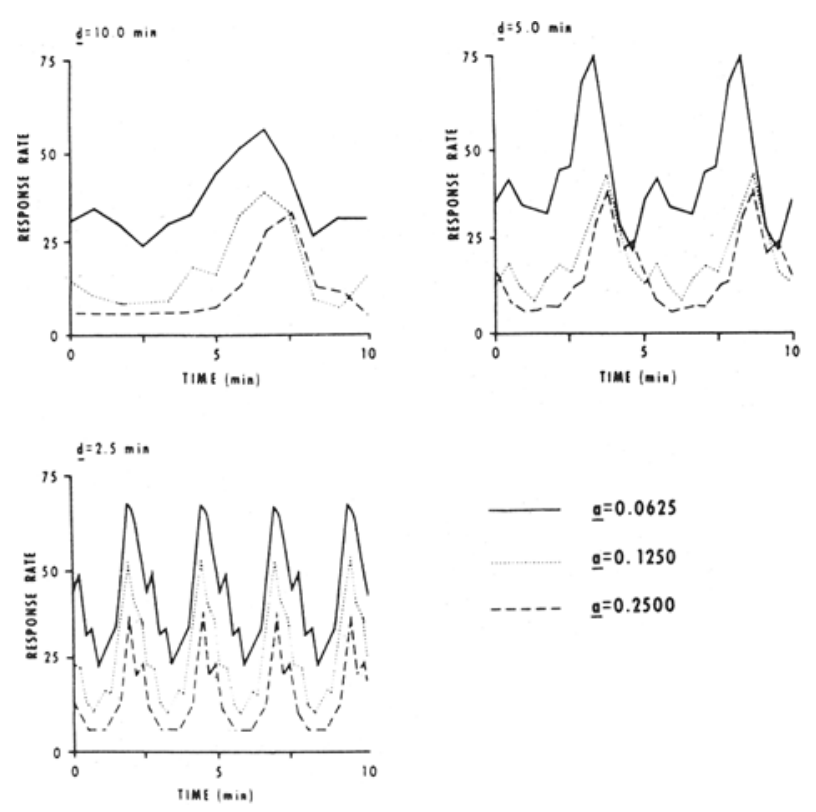

Figure 2. Illustrative response rate rhythms generated by the servo-control arrangement.

determine response rate per interval measures. By this method, it is possible to plot response-rate graphs on a time line and thus compare response rate rhythms generated by sinusoidal feedback functions having different locations, amplitudes, and/or periods. Figure 2, for example, shows illustrative leverpress response rate rhythms generated in a single rat during 4-h trials wherein parameter $b$ was equal to $a$. These graphs show that response rate rhythms generated by the servo-control arrangement have locations which covary inversely with a, and periodicities which covary directly with d.

We also determine a measure of the subject's "performance efficiency" during a trial. That is, if the animal responds chiefly at times which correspond to peaks of the sinusoid (Equation 1), then the ratio of reinforcers received to responses emitted $(S / R)$ will approximate the maximum value of the sinusoid. Conversely, if the animal responds chiefly at times which correspond to troughs of the sinusoid, then $S / R$ will approximate the minimum value of the sinusoid. Between these extremes, the animal may respond chiefly at random times and $\mathrm{S} / \mathrm{R}$ then will approximate the average value of the sinusoid. A measure of performance efficiency (eff) relative to timewise random responding thus may be defined:

$$
\text { eff }=\frac{S / R}{F(t) \text { ave }} .
$$

Figure 3 shows average eff ratios generated by the sinusoidal feedback arrangements described in connection with Figure 2: whereas an increase of a produces a decrease in eff, an increase of $d$ produces an increase in eff.

The data reported above were obtained with a Hewlett-Packard 2114-A laboratory computer programmed in BASIC. Since this early work, the servo-control system has been expanded to incorporate 10 independently manipulable variables, and we now use a PDP-12 laboratory computer programmed in FORTRAN. ${ }^{1}$ Although a great amount of "dose-response" type of research remains to be done in connection with each of the 10 variables, our results to date have implications for the study of specific issues in several areas. To illustrate, consider how a serwo analysis of behavior rhythms might contribute to amelioration and/or prevention of developmental disabilities in premature, low-birthweight human infants.

Small, premature (and some other at-risk) human neonates do not exhibit temporally regular biological rhythms as do healthy babies. On the contrary, such processes as brain electrical activity and motility in premature infants tend to be timewise erratic or relatively constant. Barring other complications, rhythmical temporal patterns emerge gradually and subsequent disability is unlikely; with complications, however, arhythmical temporal patterns continue and the likelihood of subsequent disability increases. One possible application of servo control therefore is in an attempt to normalize behavior rhythms in such babies. Undoubtedly, such an effort will not be easy. To begin, an effective feedback stimulus-perhaps vibration, rocking, or stroking-will have to be discovered. But such an effort will not be impossible either. In the laboratory, for example, physiological rhythms may be monitored when behavior rhythms are servo-controlled to determine interrelationships between them; or, using biofeedback technology,

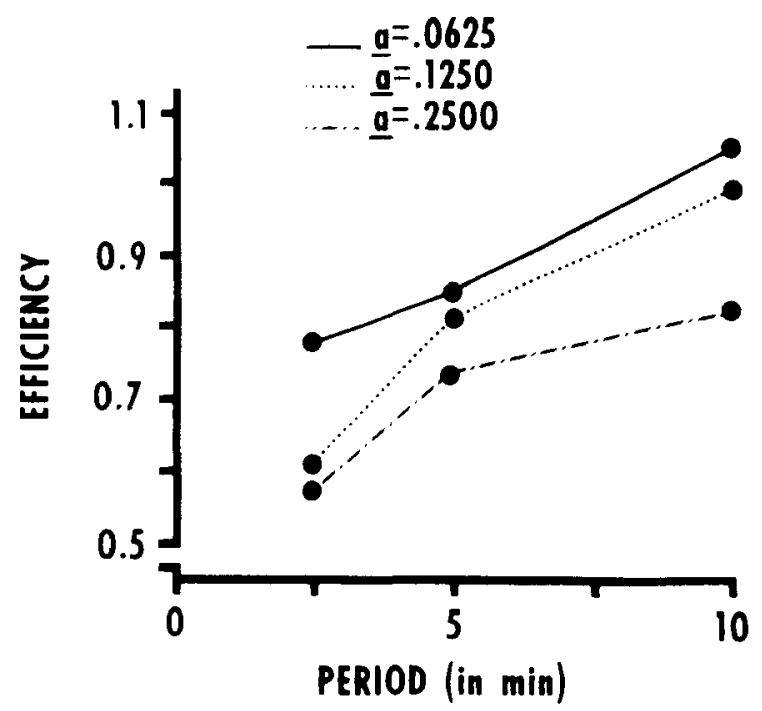

Figure 3. Average performance efficiency ratios generated by nine sinusoidal command signals. 
servo control of physiological rhythms may be studied directly. Similarly, the ontogenesis of normal and abnormal biorhythms may be investigated systematically by longitudinal study of rhythms in offspring of adult animals maintained in servo-controlled environments. Granted, each such application, and other applications too, may have special peripheral interface requirements and/or special data processing requirements. These problems, however, are external to the servo-control arrangement, and thus it is available to a wide range of issues.

\section{REFERENCE}

Bellville, J. W., Fleischli, G., \& Attura, G. Servo control of inhaled carbon dioxide. Journal of Applied Physiology' 1968, 24, 414-415.

\section{NOTE}

1. Documentation is available from the authors. 